\title{
JOSÉ CARRILLO
}

\section{GIANNI GILARDI}

\section{La vitesse de propagation dans le problème de la digue}

Annales de la faculté des sciences de Toulouse $5^{e}$ série, tome 11, no 3 (1990), p. 7-28

<http://www.numdam.org/item?id=AFST_1990_5_11_3_7_0>

(C) Université Paul Sabatier, 1990, tous droits réservés.

L'accès aux archives de la revue «Annales de la faculté des sciences de Toulouse » (http://picard.ups-tlse.fr/ annales/) implique l'accord avec les conditions générales d'utilisation (http://www.numdam.org/conditions). Toute utilisation commerciale ou impression systématique est constitutive d'une infraction pénale. Toute copie ou impression de ce fichier doit contenir la présente mention de copyright.

\section{NumDam}

Article numérisé dans le cadre du programme Numérisation de documents anciens mathématiques http://www.numdam.org/ 


\title{
La vitesse de propagation dans le problème de la digue
}

\author{
José Carrillo ${ }^{(1)}$ and Gianni Gilardi ${ }^{(2)}$
}

\begin{abstract}
RÉSUMÉ. - Dans ce travail nous étudions la vitesse de propagation de la frontière libre dans le problème de la digue. Nous démontrons que la frontière libre de la zone saturée $(\chi=1)$ a une vitesse finie de propagation, ce qui implique que la vitesse de propagation de la pression est finie si le milieu est non saturé. Si le milieu est saturé la vitesse de propagation de la pression peut être infinie.
\end{abstract}

Abstract. - In this paper we study the speed of propagation of the free boundary of the dam problem. We prove that the free boundary of the saturated part $(\chi=1)$ has a finite speed of propagation, what implies that the speed of propagation of the pressure is finite when the medium is not saturated. When the medium is saturated the speed of propagation of the pressure can be infinite.

\section{Introduction}

Dans ce travail nous présentons un résultat de vitesse finie de propagation pour la solution du problème d'évolution de la digue.

Des résultats de ce type sont bien connus pour l'équation des milieux poreux, à savoir

$$
\partial_{t} \beta(u)-\Delta u=0
$$

si $\beta$ n'est pas trop régulier, par exemple si $\beta(u)=u^{\alpha}$ avec $0<\alpha<1$ (voir $[1],[3]$ et les références de ces travaux). L'équation de la digue est un cas

(1) Departamento de Matemática Aplicada, Universidad Complutense de Madrid (Espagne)

(2) Dipartimento di Matematica, Università di Pavia (Italie).

Soutenu par le Ministero della Pubblica Istruzione et par l'Istituto di Analisi Numerica del C.N.R. di Pavia (Italie) 
limite d'une équation du type (0.1) avec un terme de convection dû à la gravité; concrètement

$$
\begin{cases}\partial_{t} \chi-\operatorname{div}(\nabla u+\chi e)=0 & \operatorname{dans} \Omega \times(0, T) \\ 0 \leq \chi \leq 1 & \operatorname{dans} \Omega \times(0, T) \\ (1-\chi) u=0 & \text { dans } \Omega \times(0, T)\end{cases}
$$

où $\Omega$ est un ouvert borné lipschitzien de $\mathbb{R}^{n}$ et $e$ est le vecteur vertical unitaire orienté positivement. Le problème complet est obtenu en ajoutant à $(0.2)$ les conditions initiales et les conditions aux limites qui sont de deux types :

$$
\begin{gathered}
\begin{cases}u=g & \text { sur } \Gamma_{D} \times(0, T) \\
(\nabla u+\chi e) \cdot \nu \leq 0 & \text { sur } \Gamma_{D} \times(0, T) \cap\{u=0\}\end{cases} \\
(\nabla u+\chi e) \cdot \nu=0 \quad \text { sur } \Gamma_{N} \times(0, T),
\end{gathered}
$$

où $\Gamma_{D}$ est la partie perméable de $\partial \Omega$, que nous supposons de mesure positive. $\Gamma_{N}$ est la partie imperméable et $\nu$ est le vecteur normal unitaire sur $\partial \Omega$ orienté vers l'extérieur; de plus $g$ est une fonction donnée non négative.

La condition initiale est

$$
\chi(x, 0)=\chi^{0}(x) \quad \operatorname{dans} \Omega
$$

où $\chi^{0}$ est une fonction donnée vérifiant $0 \leq \chi^{0} \leq 1$.

Du point de vue physique $u$ et $g$ représentent des pressions normalisées (la pression atmosphérique étant égale à 0$), \chi$ et $\chi^{0}$ représentent des saturations du fluide et $-(\nabla u+\chi e)$ représente la vitesse d'écoulement du fluide dans le milieu poreux (loi de Darcy). L'interprétation physique de (0.4) est donc qu'il n'y a pas de flux à travers $\Gamma_{N}$, tandis que le flux peut être positif sur la partie de $\Gamma_{D}$ où la pression est nulle.

La formulation faible de (0.2)-(0.4) est la suivante :

$$
\begin{gathered}
u \in L^{2}\left(0, T ; H^{1}(\Omega)\right), u \geq 0 \text { dans } Q, \\
u=g \text { sur } \Sigma_{D}, \\
\chi \in L^{\infty}(Q), 0 \leq \chi \leq 1 \text { et } u(1-\chi)=0 \text { dans } Q, \\
\left\{\begin{array}{l}
\int_{Q}\left(-\chi \partial_{t} v+(\nabla u+\chi e) \cdot \nabla v\right) \leq 0 \\
\forall v \in H^{1}(Q) \text { telle que } v \geq 0 \text { sur } \Sigma_{D}, v=0 \text { sur } \Sigma_{D} \cap\{g>0\}, \\
v(x, 0)=v(x, T)=0 \text { dans } \Omega .
\end{array}\right.
\end{gathered}
$$


Dans la formulation faible du problème ainsi que dans le reste de ce travail nous utilisons les notations suivantes :

$$
\begin{aligned}
Q & =\Omega \times(0, T) ; \quad \Sigma_{D}=\Gamma_{D} \times(0, T) ; \quad \Sigma_{N}=\Gamma_{N} \times(0, T) \\
V & =\left\{v \in H^{1}(\Omega) / v=0 \text { sur } \Gamma_{D}\right\}
\end{aligned}
$$

Remarquons que (0.9) implique

$$
\left|\int_{Q} \chi \partial_{t} v\right| \leq c\|v\|_{L^{2}(0, T ; V)}
$$

pour toute fonction $v \in \mathcal{D}(0, T ; V)$, ce qui entraîne

$$
\partial_{t} \chi \in L^{2}\left(0, T ; V^{\prime}\right)
$$

et

$$
\left\langle\partial_{t} \chi, v\right\rangle+\int_{Q}(\nabla u+\chi e) \cdot \nabla v=0, \quad \forall v \in L^{2}(0, T ; V) ;
$$

en particulier (0.5) a un sens.

Sous des hypothèses convenables le problème (0.5)-(0.9) a été étudié dans de nombreux travaux (voir [2], [4], [5], [6], [7], [8] et [9]) et on connaît en particulier des théorèmes d'existence ainsi que d'unicité.

Le plan de ce travail est le suivant : dans la section 1 nous donnons quelques propriétés supplémentaires de la solution; dans la section 2 nous donnons un théorème de comparaison entre solutions et sursolutions; enfin dans la section 3 nous étudions le problème de la vitesse de propagation de la solution et nous faisons quelques remarques complémentaires.

\section{Quelques propriétés de la solution}

Dans cette section nous présentons quelques propriétés supplémentaires de la solution du problème (0.5)-(0.9). En premier lieu nous démontrons un résultat technique.

LEMME 1.1. - Soit $(u, \chi)$ une solution de (0.5)-(0.9) et soient $\varphi \in$ $L^{2}(0, T ; V)$ telle que $\varphi(1-\chi)=0$ dans $Q$. Alors

$$
\left\langle\partial_{t} \chi, \varphi\right\rangle=0 .
$$


Démonstration. - Soient $\delta>0$ et $\psi \in \mathcal{D}(0, T)$ avec supp $\psi \subset[\delta, T-\delta]$ et $\psi \geq 0$. Nous supposerons d'abord que $\varphi \geq 0$. Soit $v=\varphi \psi$, alors pour tout $\tau \in(-\delta, \delta)$ la fonction $v_{\tau}(x, t)=v(x, t-\tau)$ appartient à $L^{2}(0, T ; V)$ et, d'après $(0.13)$ nous avons

$$
-\int_{Q}(\nabla u+\chi e) \cdot \nabla v_{\tau} \mathrm{d} x \mathrm{~d} t=\left\langle\partial_{t} \chi, v_{\tau}\right\rangle .
$$

En changeant $t$ par $t+\tau$ nous obtenons

$-\int_{Q}(\nabla u(x, t+\tau)+\chi(x, t+\tau) e) \cdot \nabla v(x, t) \mathrm{d} x \mathrm{~d} t=\partial_{\tau}\langle\chi(x, t+\tau), v(x, t)\rangle ;$

d'où la fonction $\tau \rightarrow P(\tau)=\langle\chi(x, t+\tau), v(x, t)\rangle$ appartient à $C^{1}(-\delta, \delta)$. Comme

$$
\begin{aligned}
P(\tau) & =\int_{Q} \chi(x, t+\tau) v(x, t) \mathrm{d} x \mathrm{~d} t \leq \\
& \leq \int_{Q} v(x, t) \mathrm{d} x \mathrm{~d} t=\int_{Q} \chi(x, t) v(x, t) \mathrm{d} x \mathrm{~d} t=P(0),
\end{aligned}
$$

pour tout $\tau \in(-\delta, \delta)$, nous déduisons que $P^{\prime}(0)=0$, i.e.

$$
\int_{Q}(\nabla u+\chi e) \cdot \nabla v=0
$$

Donc, d'après $(0.13)$

$$
\left\langle\partial_{t} \chi, v\right\rangle=0
$$

Il suffit de choisir une suite $\delta_{m}$ et une suite $\psi_{m}$ telles que les $v_{m}=\varphi \psi_{m}$ convergent vers $\varphi$ dans $L^{2}(0, T ; V)$ pour avoir (1.1).

Pour une fonction $\varphi$ dont le signe n'est pas déterminé le résultat est encore vrai pour $\varphi^{+}$et $\varphi^{-}$donc pour $\varphi$. $\square$

Remarque 1.2. - Le résultat du lemme 1.1 est encore vrai pour les fonctions de $L^{2}\left(0, T ; H_{0}^{1}(\Omega)\right)$ quand $(u, \chi)$ vérifie seulement $(0.2)$. La démonstration antérieure est encore valable.

Proposition 1.3. - Sig est bornée sur $\Sigma_{D}$ alors la solution $(u, \chi)$ de (0.5)-(0.9) est bornée.

Démonstration. - Si $g$ est bornée nous pouvons fixer $h \in \mathbb{R}$ tel que la fonction $w(x)=h-x \cdot e$ soit positive sur $\Omega$ et $w(x) \geq g(x, t)$ sur $\Gamma_{D}$ pour 
tout $t \in(0, T)$. Soit alors $v(x, t)=(u(x, t)-w(x))^{+}$, d'après $(0.9)$ et le lemme 1.1 , nous obtenons

$$
0=\int_{Q}(\nabla u+\chi e) \cdot \nabla v=\int_{Q}(\nabla u+e) \cdot \nabla v=\int_{Q} \nabla v \cdot \nabla v,
$$

d'où $v=0$.

Proposition 1.4. - Soient $u \in L^{2}\left(0, T ; H^{1}(\Omega)\right)$ et $\chi \in L^{\infty}(Q)$ satisfaisant (0.2). Alors

$$
\Delta u \geq 0 \quad \text { et } \quad \partial_{t} \chi-\operatorname{div}(\chi e) \geq 0 \quad \text { dans } Q .
$$

Démonstration. - Soit $v \in \mathcal{D}(Q), v \geq 0$, alors pour tout $\epsilon>0$ la fonction $w=\min (u / \epsilon, v)$ est une fonction de $L^{2}\left(0, T ; H_{0}^{1}(\Omega)\right)$ qui vérifie $(1-\chi) w=0$. D'après le lemme 1.1 et la remarque 1.2 nous avons

$$
\int_{Q}(\nabla u+\chi e) \cdot \nabla w=0
$$

D'autre part, d'après le théorème de la divergence

$$
\int_{Q} \chi e \cdot \nabla w=0
$$

d'où

$$
\int_{Q} \nabla u \cdot \nabla w=0
$$

et donc

$$
\int_{Q \cap\{u>\epsilon v\}} \nabla u \cdot \nabla w=-\frac{1}{\epsilon} \int_{Q \cap\{u \leq \epsilon v\}}|\nabla u|^{2} .
$$

À la limite on obtient

$$
\int_{Q} \nabla u \cdot \nabla v \leq 0
$$

d'où le résultat.

Remarque 1.5. - Le résultat précédent a évidemment un caractère local, il est donc encore valable si on suppose seulement $u \in L_{\text {loc }}^{2}\left(0, T ; H_{\text {loc }}^{1}(\Omega)\right)$. De même l'hypothèse que $\Omega$ soit borné n'est pas nécessaire.

Une conséquence de $(0.12)$ est que $\chi \in C^{0}\left([0, T] ; V^{\prime}\right)$. D'autre part, $\chi \in L^{\infty}\left(0, T ; L^{\infty}(\Omega)\right)$. Le résultat suivant montre une continuité plus forte. 
Proposition 1.6. - Soit $(u, \chi)$ une solution de (0.5)-(0.9). Alors $\chi \in$ $C^{0}\left([0, T] ; L^{p}(\Omega)\right)$ pour tout $p \geq 1$, fini.

Démonstration. - En tenant compte de (0.12) et du fait que $\chi$ est bornée on déduit aisément que pour tout $t$ fixé nous avons

$$
\begin{array}{r}
\chi(x, t+h) \rightarrow \chi(x, t) \text { faiblement dans } L^{p}(\Omega) \text { quand } h \rightarrow 0, \\
\text { ceci pout tout } p \text { tel que } 1<p<\infty .
\end{array}
$$

Considérons, maintenant, $p>1$ fini, et soit $\Omega_{h}=\{x \in \Omega \mid d(x, \partial \Omega)>h\}$, alors

$$
\begin{aligned}
& \left|\int_{\Omega}\left(\chi^{p}(x, t)-\chi^{p}(x, t+h)\right)\right| \leq\left|\int_{\Omega} \chi^{p}(x, t)-\int_{\Omega_{h}} \chi^{p}(x+e h, t)\right|+ \\
& \quad+\left|\int_{\Omega_{h}}\left(\chi^{p}(x+e h, t)-\chi^{p}(x, t+h)\right)\right|+\left|\int_{\Omega} \chi^{p}(x, t+h)-\int_{\Omega_{h}} \chi^{p}(x, t+h)\right| .
\end{aligned}
$$

Il est évident que la première et la dernière valeurs absolues (à droite de l'inégalité) convergent vers 0 quand $h \rightarrow 0$. Pour ce qui est de la seconde valeur absolue, en tenant compte de (1.2) et de l'inégalité $\left|a^{p}-b^{p}\right| \leq p|a-b|$, $\forall a, b \in[0,1]$, nous pouvons écrire

$$
\left|\int_{\Omega_{h}}\left(\chi^{p}(x+e h, t)-\chi^{p}(x, t+h)\right)\right| \leq p \int_{\Omega_{h}}(\chi(x, t+h)-\chi(x+e h, t)),
$$

et donc (1.3) donne aisément

$$
\left|\int_{\Omega_{h}}\left(\chi^{p}(x+e h, t)-\chi^{p}(x, t+h)\right)\right| \rightarrow 0 \quad \text { quand } h \rightarrow 0 .
$$

D'où

$$
\int_{\Omega}\left(\chi^{p}(x, t)-\chi^{p}(x, t+h)\right) \rightarrow 0 \quad \text { quand } h \rightarrow 0 .
$$

De (1.3) et (1.4) nous pouvons déduire que

$$
\begin{gathered}
\chi(x, t+h) \rightarrow \chi(x, t) \text { dans } L^{p}(\Omega) \text { quand } h \rightarrow 0, \\
\text { pout tout } p>1 \text { fini, }
\end{gathered}
$$

et donc

$$
\begin{aligned}
& \chi(x, t+h) \rightarrow \chi(x, t) \text { dans } L^{p}(\Omega) \text { quand } h \rightarrow 0, \\
& \text { pout tout } p \text { tel que } 1 \leq p<\infty,
\end{aligned}
$$

d'où le résultat. 


\section{Résultats de comparaison}

Dans cette section nous commençons par donner quelques résultats techniques relatifs à la solution du problème $(0.5)-(0.9)$.

Lemme 2.1. - Soit $(u, \chi)$ une solution de (0.5)-(0.9). Alors nous avons

$$
\begin{gathered}
\int_{Q}\left(\left(\nabla u+(\chi-\lambda)^{+} e\right) \cdot \nabla v-(\chi-\lambda)^{+} \partial_{t} v\right) \leq 0 \\
\int_{Q}\left(\nabla u \cdot \nabla \min \left(\frac{u}{\epsilon}, v\right)+(\chi-\lambda)^{+} e \cdot \nabla v-(\chi-\lambda)^{+} \partial_{t} v\right) \leq \\
\leq \lim _{\epsilon \rightarrow 0} \frac{1}{\epsilon} \int_{\{u \leq \epsilon v\}}|\nabla u|^{2} \\
\int_{Q} \nabla u \cdot \nabla \min \left(\frac{(u-k)^{+}}{\epsilon}, v\right)=0
\end{gathered}
$$

pour tout $\lambda \in[0,1], \epsilon>0, k \geq 0$ et pour toute fonction $v \in \mathcal{D}(Q)$ non négative.

Démonstration. - D'après (1.2) la fonction $t \rightarrow \chi(x-t e, t)$ est localement croissante pour presque tout $x$ fixé. C'est donc encore vrai pour les fonctions $t \rightarrow(\chi(x-t e, t)-\lambda)^{ \pm}$, où $\xi^{+}=\max (\xi, 0)$ et $\xi^{-}=\min (\xi, 0)$. Cela implique

$$
\left(\partial_{t}-\partial_{y}\right)(\chi-\lambda)^{ \pm} \geq 0
$$

où $y=x \cdot e$. Alors

$$
\begin{aligned}
\left(\partial_{t}-\partial_{y}\right)(\chi-\lambda)^{+} & =\left(\partial_{t}-\partial_{y}\right)(\chi-\lambda)-\left(\partial_{t}-\partial_{y}\right)(\chi-\lambda)^{-} \leq \\
& \leq\left(\partial_{t}-\partial_{y}\right)(\chi-\lambda)=\left(\partial_{t}-\partial_{y}\right) \chi
\end{aligned}
$$

d'où

$$
\int_{Q}(\chi-\lambda)^{+}\left(\partial_{y}-\partial_{t}\right) v \leq \int_{Q} \chi\left(\partial_{y}-\partial_{t}\right) v
$$

et (2.1) est alors immédiat.

Démontrons maintenant (2.3) en choisissant $w=\min \left((u-k)^{+} / \epsilon, v\right)$ comme fonction test. Puisque $\chi=1$ où $w>0$ nous déduisons

$$
\int_{Q} \chi e \cdot \nabla w=\int_{Q} e \cdot \nabla w=0
$$


D'autre part, d'après le lemme $1.1,\left\langle\partial_{t} \chi, w\right\rangle=0$. Donc (0.9) donne (2.3). En particulier, en prenant $k=0$ :

$$
0=\int_{Q} \nabla u \cdot \nabla \min \left(\frac{u}{\epsilon}, v\right)=\int_{\{u \leq \epsilon v\}} \frac{|\nabla u|^{2}}{\epsilon}+\int_{\{u>\epsilon v\}} \nabla u \cdot \nabla v,
$$

d'où

$$
\int_{Q} \nabla u \cdot \nabla v=-\lim _{\epsilon \rightarrow 0} \frac{1}{\epsilon} \int_{\{u \leq \epsilon v\}} \frac{|\nabla u|^{2}}{\epsilon} ;
$$

(2.2) se déduit alors de (2.1).

Donnons la définition de sursolution locale.

DÉfinition 2.4. - Un couple $(u, \chi)$ avec $u \in L^{2}\left(0, T ; H^{1}(B)\right)$ et $\chi \in L^{\infty}(B \times(0, T)) \cap C^{0}\left([0,1] ; L^{1}(B)\right)$, où $B$ est une boule de $\Omega$, est une sursolution (locale) si et seulement si

$$
\begin{gathered}
u \geq 0, \quad 0 \leq \chi \leq 1, \quad u(1-\chi)=0 \text { dans } Q^{\prime}=B \times(0, T) \\
\int_{Q^{\prime}}\left(-\chi \partial_{t} v+(\nabla u+\chi e) \cdot \nabla v\right) \geq 0, \quad \forall v \in H_{0}^{1}\left(Q^{\prime}\right), v \geq 0 \\
\int_{Q^{\prime}} \nabla u \cdot \nabla v \leq 0, \quad \forall v \in L^{2}\left(0, T ; H_{0}^{1}(B)\right), v \geq 0 .
\end{gathered}
$$

Remarque 2.5. - $\mathrm{Si}(u, \chi)$ est une sursolution et si $u>0$ dans un ouvert $Q^{\prime \prime} \subset Q^{\prime}$ alors $\Delta u=0$ dans $Q^{\prime \prime}$.

Remarque 2.6. - En ajoutant (2.5) à (2.6) nous avons

$$
\partial_{t} \chi-\operatorname{div}(\chi e) \geq 0 \text {. }
$$

Lemme 2.7. - Soit $(u, \chi)$ une sursolution dans $Q^{\prime}=B \times(0, T)$, où $B$ est une boule de $\Omega$. Alors

$$
\int_{Q^{\prime}}\left(\nabla u \cdot \nabla \min \left(\frac{(k-u)^{+}}{\epsilon}, v\right)+(\chi-\lambda)^{-}\left(\partial_{y}-\delta_{t}\right) v\right) \geq 0
$$

pour toute fonction $v \in \mathcal{D}\left(Q^{\prime}\right), v$ non négative et pour $k \geq 0$ et $\lambda \in H(k)$ où $H$ est le graphe maximal monotone associé à la fonction de Heaviside. 
Démonstration. - Nous considérons deux cas : $k=0$ et $k>0$.

Si $k=0,(2.8)$ se réduit à

$$
\int_{Q^{\prime}}(\chi-\lambda)^{-}\left(\partial_{y}-\partial_{t}\right) v \geq 0
$$

qui se démontre avec un argument identique à celui de la démonstration de (2.1), en tenant compte de la remarque 2.6.

Soit maintemant $k>0$. De (2.5) et du fait que $v$ est une fonction non négative nulle sur $\partial Q^{\prime}$ nous déduisons que

$$
\int_{Q^{\prime}}\left(\nabla u \cdot \nabla v+(\chi-1)\left(\partial_{y}-\partial_{t}\right) v\right) \geq 0 .
$$

D'autre part (2.6) implique que l'opérateur $v \rightarrow \int_{Q^{\prime}} \nabla u \cdot \nabla v$ est décroissant, d'où

$$
\int_{Q^{\prime}} \nabla u \cdot \nabla v \leq \int_{Q^{\prime}} \nabla u \cdot \nabla \min \left(\frac{(k-u)^{+}}{\epsilon}, v\right) .
$$

De (2.9) et (2.10) nous déduisons le résultat.

THÉORÈME 2.8. - Soient $(u, \chi)$ une solution de (0.5)-(0.9) et $(\bar{u}, \bar{\chi})$ une sursolution dans $Q_{R}=B_{R} \times(0, T)$ où $B_{R}$ est une boule de $\Omega$. Nous supposons qu'il existe $R^{\prime} \in(0, R)$ telle que $u<\bar{u}$ dans $\left(B_{R}-B_{R^{\prime}}\right) \times(0, T)$ et $\chi(\cdot, 0) \leq \bar{\chi}(\cdot, 0)$ dans $B_{R}$. Alors

$$
u \leq \bar{u} \quad \text { et } \quad \chi \leq \bar{\chi} \quad \text { dans } Q_{R}
$$

Démonstration. - Nous considérons $(u, \chi)$ et $\bar{u}, \bar{\chi}$ comme des fonctions définies sur $Q_{R} \times Q_{R}$, c'est-à-dire :

$$
\begin{aligned}
& (u, \chi):(x, t, \xi, \tau) \rightarrow(u(x, t), \chi(x, t)) \\
& (\bar{u}, \bar{\chi}):(x, t, \xi, \tau) \rightarrow(\bar{u}(\xi, \tau), \bar{\chi}(\xi, \tau)) .
\end{aligned}
$$

Soit $v \in \mathcal{D}\left(Q_{R} \times Q_{R}\right)$ une fonction non négative. En appliquant (2.2) avec $\lambda=\bar{\chi}(\xi, \tau)$ nous obtenons

$$
\begin{gathered}
\int_{Q_{R}}\left(\nabla_{x} u \cdot \nabla_{x} \min \left(\frac{u}{\epsilon}, v\right)+(\chi-\bar{\chi})^{+} e \cdot \nabla_{x} v-(\chi-\bar{\chi})^{+} \partial_{t} v\right) \mathrm{d} x \mathrm{~d} t \leq \\
\leq \lim _{\epsilon \rightarrow 0} \frac{1}{\epsilon} \int_{\{u \leq \epsilon v\}}\left|\nabla_{x} u\right|^{2} \mathrm{~d} x \mathrm{~d} t, \quad \\
-15-
\end{gathered}
$$


José Carrillo et Gianni Gilardi

pour presque tout $(\xi, \tau) \in Q_{R}$ tel que $\bar{u}(\xi, \tau)=0$. En appliquant (2.3) avec $k=\bar{u}(\xi, \tau)$ nous avons

$$
\int_{Q_{R}} \nabla_{x} u \cdot \nabla_{x} \min \left(\frac{(u-\bar{u})^{+}}{\epsilon}, v\right) \leq 0,
$$

pour presque tout $(\xi, \tau) \in Q_{R}$ tel que $\bar{u}(\xi, \tau)>0$. En intégrant (2.12) sur $\left\{(\xi, \tau) \in Q_{R} \mid \bar{u}(\xi, \tau)=0\right\}$ et $(2.13)$ sur $\left\{(\xi, \tau) \in Q_{R} \mid \bar{u}(\xi, \tau)>0\right\}$ et en sommant les deux intégrales obtenues nous avons

$$
\begin{aligned}
& \int_{Q_{R} \times Q_{R}}\left(\nabla_{x} u \cdot \nabla_{x}\right.\left.\min \left(\frac{(u-\bar{u})^{+}}{\epsilon}, v\right)+(\chi-\bar{\chi})^{+} e \cdot \nabla_{x} v-(\chi-\bar{\chi})^{+} \partial_{t} v\right) \\
& \leq \lim _{\epsilon \rightarrow 0} \frac{1}{\epsilon} \int_{\{\bar{u}=0\}} \mathrm{d} \xi \mathrm{d} \tau \int_{\{u \leq \epsilon v\}}\left|\nabla_{x} u\right|^{2} \mathrm{~d} x \mathrm{~d} t
\end{aligned}
$$

car, d'après le théorème de Lebesgue,

$$
\begin{aligned}
\lim _{\epsilon \rightarrow 0} \frac{1}{\epsilon} \int_{\{\bar{u}=0\}} \mathrm{d} \xi \mathrm{d} \tau \int_{\{u \leq \epsilon v\}}\left|\nabla_{x} u\right|^{2} \mathrm{~d} x \mathrm{~d} t= \\
=\int_{\{\bar{u}=0\}} \mathrm{d} \xi \mathrm{d} \tau \lim _{\epsilon \rightarrow 0} \frac{1}{\epsilon} \int_{\{u \leq \epsilon v\}}\left|\nabla_{x} u\right|^{2} \mathrm{~d} x \mathrm{~d} t .
\end{aligned}
$$

En intégrant maintenant $(2.8)$ sur $Q_{R}$ par rapport à $(x, t)$ avec $(k, \lambda)=$ $(u(x, t), \chi(x, t))$ nous obtenons

$$
\int_{Q_{R} \times Q_{R}}\left(\nabla_{\xi} \bar{u} \cdot \nabla_{\xi} \min \left(\frac{(u-\bar{u})^{+}}{\epsilon}, v\right)+(\bar{\chi}-\chi)^{-}\left(e \cdot \nabla_{\xi} v-\partial_{t} v\right)\right) \leq 0 .
$$

En soustrayant (2.15) de (2.14) nous avons

$$
\begin{array}{r}
\int_{Q_{R} \times Q_{R}}\left(\nabla_{x} u \cdot \nabla_{x} \min \left(\frac{(u-\bar{u})^{+}}{\epsilon}, v\right)-\nabla_{\xi} \bar{u} \cdot \nabla_{\xi} \min \left(\frac{(u-\bar{u})^{+}}{\epsilon}, v\right)\right)+ \\
\quad+\int_{Q_{R} \times Q_{R}}(\chi-\bar{\chi})^{+}\left(e \cdot\left(\nabla_{x} v+\nabla_{\xi} v\right)-\left(\partial_{t} v+\partial_{\tau} v\right)\right) \leq \\
\leq \lim _{\epsilon \rightarrow 0} \frac{1}{\epsilon} \int_{\{\bar{u}=0\}} \mathrm{d} \xi \mathrm{d} \tau \int_{\{u \leq \epsilon v\}}\left|\nabla_{x} u\right|^{2} \mathrm{~d} x \mathrm{~d} t .
\end{array}
$$

Une intégration par parties par rapport à $\xi$ nous donne

$$
\int_{Q_{R} \times Q_{R}}\left(\nabla_{\mathfrak{x}} u \cdot \nabla_{\xi} \min \left(\frac{(u-\bar{u})^{+}}{\epsilon}, v\right)\right)=0
$$

$-16-$ 
La vitesse de propagation dans le problème de la digue

et similairement

$$
-\int_{Q_{R} \times Q_{R}} \nabla_{\xi} \bar{u} \cdot \nabla_{x} \min \left(\frac{(u-\bar{u})^{+}}{\epsilon}, v\right)=0 .
$$

En sommant (2.16), (2.17) et (2.18) nous obtenons

$$
\begin{aligned}
& \int_{Q_{R} \times Q_{R}}\left(\nabla_{x} u-\nabla_{\xi} \bar{u}\right) \cdot\left(\nabla_{x} \min \left(\frac{(u-\bar{u})^{+}}{\epsilon}, v\right)+\nabla_{\xi} \min \left(\frac{(u-\bar{u})^{+}}{\epsilon}, v\right)\right) \\
& \quad+\int_{Q_{R} \times Q_{R}}(\chi-\bar{\chi})^{+}\left(e \cdot\left(\nabla_{x} v+\nabla_{\xi} v\right)-\left(\partial_{t} v+\partial_{\tau} v\right)\right) \leq \\
& \leq \lim _{\epsilon \rightarrow 0} \frac{1}{\epsilon} \int_{\{\bar{u}=0\}} \mathrm{d} \xi \mathrm{d} \tau \int_{\{u \leq \epsilon v\}}\left|\nabla_{x} u\right|^{2} \mathrm{~d} x \mathrm{~d} t .
\end{aligned}
$$

Faisons maitenant tendre $\epsilon$ vers 0 :

$$
\begin{aligned}
\lim _{\epsilon \rightarrow 0} \frac{1}{\epsilon} \int_{\{\bar{u}=0\}} \mathrm{d} \xi \mathrm{d} \tau \int_{\{u \leq \epsilon v\}} \frac{1}{\epsilon}\left|\nabla_{x} u\right|^{2} \mathrm{~d} x \mathrm{~d} t+ \\
\quad+\lim _{\epsilon \rightarrow 0} \int_{\{\bar{u}>0\}} \mathrm{d} \xi \mathrm{d} \tau \int_{\{(u-\bar{u})+\leq \epsilon v\}} \frac{1}{\epsilon}\left|\nabla_{x} u-\nabla_{\xi} \bar{u}\right|^{2} \mathrm{~d} x \mathrm{~d} t \\
\quad+\lim _{\epsilon \rightarrow 0} \int_{Q_{R}} \mathrm{~d} \xi \mathrm{d} \tau \int_{\left\{(u-\bar{u})^{+}>\epsilon v\right\}}\left(\nabla_{x} u-\nabla_{\xi} \bar{u}\right)\left(\nabla_{x}+\nabla_{\xi}\right) v \mathrm{~d} x \mathrm{~d} t \\
\quad+\int_{Q_{R} \times Q_{R}}(\chi-\bar{\chi})^{+}\left(e \cdot\left(\nabla_{x} v+\nabla_{\xi} v\right)-\left(\partial_{t} v+\partial_{\tau} v\right)\right) \\
\leq \lim _{\epsilon \rightarrow 0} \int_{\{\bar{u}=0\}} \mathrm{d} \xi \mathrm{d} \tau \int_{(u \leq \epsilon v\}}\left|\nabla_{x} u\right|^{2} \mathrm{~d} x \mathrm{~d} t .
\end{aligned}
$$

d'où, puisque la deuxième intégrale est non négative et $\nabla_{x} u-\nabla_{\xi} u=$ $\left(\nabla_{x}+\nabla_{\xi}\right)(u-\bar{u})$,

$$
\begin{aligned}
\int_{Q_{R} \times Q_{R}}\left(\left(\nabla_{x}+\right.\right. & \left.\nabla_{\xi}\right)(u-\bar{u})^{+} \cdot\left(\nabla_{x}+\nabla_{\xi}\right) v+ \\
& \left.+(\chi-\bar{\chi})^{+}\left(e \cdot\left(\nabla_{x}+\nabla_{\xi}\right) v-\left(\partial_{t}+\partial_{\tau}\right) v\right)\right) \leq 0
\end{aligned}
$$

soit encore, en tenant compte du fait que $v \in \mathcal{D}\left(Q_{R} \times Q_{R}\right), u, \bar{u}, \chi$ et $\bar{\chi}$ peuvent être prolongés arbitrairement hors de $Q_{R} \times Q_{R}$,

$$
\begin{gathered}
\int_{\mathbb{R}^{2 n+2}}\left(\left(\nabla_{x}+\nabla_{\xi}\right)(u-\bar{u})^{+} \cdot\left(\nabla_{x}+\nabla_{\xi}\right) v\right. \\
\left.+(\chi-\bar{\chi})^{+}\left(e \cdot\left(\nabla_{x}+\nabla_{\xi}\right) v-\left(\partial_{t}+\partial_{\tau}\right) v\right)\right) \leq 0 \\
-17-
\end{gathered}
$$


Cette dernière inégalité étant vraie pour toute $v \in \mathcal{D}\left(Q_{R} \times Q_{R}\right)$ non négative, nous pouvons choisir $v$ de la façon suivante. Soit $\omega$ quelconque, non négative, $\omega \in \mathcal{D}\left(Q_{R}\right)$, et soit $\delta_{0}=\operatorname{dist}\left(\operatorname{supp} \omega, \partial Q_{R}\right)$. Pour tout $\delta$ tel que $0<\delta<\delta_{0}$ soit $\rho_{\delta} \in \mathcal{D}\left(\mathbb{R}^{n+1}\right)$ avec $\rho_{\delta} \geq 0, \int \rho_{\delta}=1$ et $\operatorname{supp} \rho_{\delta} \subset B_{\delta}\left(\subset \mathbb{R}^{n+1}\right)$. Nous choisissons

$$
v(x, t, \xi, \tau)=\omega\left(\frac{x+\xi}{2}, \frac{t+\tau}{2}\right) \rho_{\delta}\left(\frac{x-\xi}{2}, \frac{t-\tau}{2}\right) .
$$

Nous introduisons le changement de variables suivant :

$$
\frac{x+\xi}{2}=y, \quad \frac{t+\tau}{2}=\eta, \quad \frac{x-\xi}{2}=z, \quad \frac{t-\tau}{2}=\zeta .
$$

De plus nous posons :

$$
\begin{array}{ll}
w(y, \eta, z, \zeta)=u(y+z, \eta+\zeta), & \theta(y, \eta, z, \zeta)=\chi(y+z, \eta+\zeta) \\
\bar{w}(y, \eta, z, \zeta)=\bar{u}(y-z, \eta-\zeta), & \bar{\theta}(y, \eta, z, \zeta)=\bar{\chi}(y-z, \eta-\zeta)
\end{array}
$$

Alors (2.19) s'écrit :

$$
\int_{\mathbb{R}^{2 n+2}} \rho_{\delta}\left(\nabla_{y}(w-\bar{w})^{+} \cdot \nabla_{y} \omega+(\theta-\bar{\theta})^{+}\left(e \cdot \nabla_{y} \omega-\partial_{\eta} \omega\right)\right) \leq 0 .
$$

En faisant tendre $\delta$ vers 0 nous obtenons :

$$
\left.\int_{\mathbb{R}^{n+1}}\left(\nabla_{y}(w-\bar{w})^{+} \cdot \nabla_{y} \omega+(\theta-\bar{\theta})^{+}\left(e \cdot \nabla_{y} \omega-\partial_{\eta} \omega\right)\right)\right|_{(z, \zeta)=(0,0)} \leq 0,
$$

c'est-à-dire, en changeant les variables,

$$
\begin{aligned}
& \int_{Q_{R}}\left(\nabla(u(x, t)-\bar{u}(x, t))^{+} \cdot \nabla \omega(x, t)+\right. \\
& \left.\quad+(\chi(x, t)-\bar{\chi}(x, t))^{+}\left(e \cdot \nabla \omega(x, t)-\partial_{t} \omega(x, t)\right)\right) \mathrm{d} x \mathrm{~d} t \leq 0,
\end{aligned}
$$

(2.20) étant vrai pour toute fonction $\omega \in \mathcal{D}\left(Q_{R}\right)$ non négative. En particulier nous choisissons $\omega(x, t)=h(x) k(t)$ où $h \in \mathcal{D}\left(B_{R}\right), h \geq 0$ dans $B_{R}$ et $k=1$ dans $B_{R^{\prime}}$, et $h \in \mathcal{D}(0, T)$ et $k \geq 0$. En tenant compte du fait que $\bar{\chi}=1 \geq \chi$ et $\bar{u}>u$ dans $B_{R}-B_{R^{\prime}} \times(0, T)$ ce qui entraîne $\left(\nabla(u-\bar{u})^{+}+(\chi-\bar{\chi})^{+} e\right) \cdot \nabla(1-\omega)=0$, nous déduisons de $(2.20)$ que

$$
\int_{Q_{R}}(\chi-\bar{\chi})^{+} k^{\prime}(t) \mathrm{d} x \mathrm{~d} t \geq 0
$$


c'est-à-dire

$$
\int_{0}^{T}\left(\int_{B_{R}}(\chi-\bar{\chi})^{+} \mathrm{d} x\right) k^{\prime}(t) \mathrm{d} t \geq 0
$$

ce qui montre que la fonction $t \rightarrow \int_{B_{R}}(\chi-\bar{\chi})^{+} \mathrm{d} x$ est décroissante dans $(0, T)$.

Puisque $\chi, \bar{\chi} \in C^{0}\left([0, T] ; L^{1}\left(B_{R}\right)\right)$ et donc

$$
(\chi-\bar{\chi})^{+} \in C^{0}\left([0, T] ; L^{1}\left(B_{R}\right)\right),
$$

nous déduisons que

$$
\int_{B_{R}}(\chi(\cdot, t)-\bar{\chi}(\cdot, t))^{+} \mathrm{d} x \leq \int_{B_{R}}(\chi(\cdot, 0)-\bar{\chi}(\cdot, 0))^{+} \mathrm{d} x=0
$$

pour tout $t \in(0, T)$, et donc

$$
\chi \leq \bar{\chi} \text { dans } Q_{R}
$$

Alors (2.20) devient simplement

$$
\int_{Q_{R}} \nabla(u-\bar{u})^{+} \cdot \nabla \omega \leq 0
$$

pour toute $\omega \in \mathcal{D}\left(Q_{R}\right)$ non négative, et donc, par densité, pour toute $\omega \in L^{2}\left(0, T ; H_{0}^{1}\left(B_{R}\right)\right)$ non négative. En particulier

$$
\int_{Q_{R}}\left|\nabla(u-\bar{u})^{+}\right|^{2} \leq 0
$$

d'où la conclusion.

\section{Vitesse de propagation}

Dans cette section nous démontrons que le problème (0.5)-(0.9) a la propriété de vitesse de propagation finie (au sens du théorème 3.5 ). Pour cela nous construisons une sursolution ad hoc (lemme 3.1 et proposition 3.4) afin d'appliquer le théorème 2.8. Le reste de la section est consacré à des remarques supplémentaires qui montrent en particulier que le théorème 3.5 est optimal. 
Lemme 3.1. - Soient $M>0, R>0$ et $c \in[0,1$ [ donnés. Alors il existe $T_{*}>0$, unique, tel que le problème de Cauchy

$$
\left\{\begin{array}{l}
r \in C^{0}\left(\left[0, T_{*}\right]\right) \cap C^{1}\left(0, T_{*}\right), \quad 0<r(t)<R \quad \text { pour tout } t \in\left(0, T_{*}\right), \\
r^{\prime}(t)=-1-\frac{M}{1-c} r^{1-n}(t)\left(\int_{r(t)}^{R} \tau^{1-n} \mathrm{~d} \tau\right)^{-1} \quad \text { pour tout } t \in(0, T), \\
r(0)=R
\end{array}\right.
$$

ait une solution maximale unique.

Démonstration. - Soit $\psi$ vérifiant

$$
\left\{\begin{array}{l}
\psi^{\prime}(s)=-\frac{\int_{s}^{R} \tau^{1-n} \mathrm{~d} \tau}{\int_{s}^{R} \tau^{1-n} \mathrm{~d} \tau+(M /(1-c)) s^{1-n}} \quad \text { pour } 0<s<R, \\
\psi(R)=0,
\end{array}\right.
$$

et soit $T_{*}=\psi(0)$. Alors $-1<\psi^{\prime}<0$ sur $(0, R)$ et donc $\psi$ est inversible de $(0, R)$ dans $\left(0, T_{*}\right)$. On vérifie aisément que $r=\psi^{-1}$ est solution de (3.1) sur $\left(0, T_{*}\right)$; de plus $r$ est maximale car $r\left(T_{*}\right)=0$ et $r^{\prime} \leq-1$ sur $\left(0, T_{*}\right)$, donc $r$ n'admet pas de prolongement régulier non négatif.

Supposons maintenant que $r$ soit une solution maximale de (3.1) sur un intervalle $\left(0, T^{\prime}\right)$. Alors $r^{\prime} \leq-1$ sur $\left(0, T^{\prime}\right)$ et, nécessairement, $r\left(T^{\prime}\right)=0$, sinon nous aurions $r\left(T^{\prime}\right)>0$ et $r$ serait prolongeable au delà de $T^{\prime}$; donc $r$ est inversible de $\left(0, T^{\prime}\right)$ dans $(0, R)$, et son inverse vérifie (3.2) avec $\psi(0)=T^{\prime}$. L'unicité de la solution de (3.2) implique alors que $T^{\prime}=T_{*}$ et que $r$ est unique.

Remarque 3.2. - Les intégrales de (3.2) peuvent être calculées pour les différents cas $n=1, n=2, n \geq 3$; le calcul de $\psi$ se réduit à une intégration supplémentaire. Par exemple, dans le cas $n=1$ nous obtenons

$$
\psi(s)=R-s-\frac{M}{1-c}\left(\log \frac{M}{1-c} \log \left(R-s+\frac{M}{1-c}\right)\right)
$$

qui montre que $\psi$ est régulière pour $s \rightarrow 0^{+}$et que $\psi^{\prime}\left(0^{+}\right)<0$, c'est-à-dire que $r$ est régulière pour $t \rightarrow T_{*}^{-}$. Par contre on peut voir que $r^{\prime}\left(T_{*}^{-}\right)=-\infty$ si $n>1$.

Remarque 3.3. - Dans ce qui suit il sera utile, et en tout cas intéressant, de connaître le comportement asymptotique, quand $t \rightarrow 0$, de la fonction $r$ 
donnée par le lemme 3.1. Avec la notation de (3.2) nous avons pour $s \rightarrow R:$

$$
\begin{aligned}
\psi^{\prime}(s) & \approx-\frac{R^{n-1} \int_{s}^{R} \tau^{1-n} \mathrm{~d} \tau}{R^{n-1} \int_{s}^{R} \tau^{1-n} \mathrm{~d} \tau+(M /(1-c))} \approx \\
& \approx-\frac{R-s}{R-s+(M /(1-c))} \approx-\frac{1-c}{M}(R-s),
\end{aligned}
$$

d'où l'on obtient $\psi(s) \approx((1-c) / 2 M)(R-s)^{2}$. Donc, pour tout $t \rightarrow 0$, nous déduisons que $t \approx((1-c) / 2 M)(R-r(t))^{2}$, d'où

$$
r(t) \approx R-\left(\frac{2 M t}{(1-c)}\right)^{\frac{1}{2}} \quad \text { quand } t \rightarrow 0 .
$$

Proposition 3.4. - Soient $M>0, R>0$ et $c \in[0,1[$ donnés et soit $r$ le prolongement trivial de la fonction donnée par le lemme 3.1. Alors le couple $(u, \chi)$ donné par les relations suivantes est une sursolution dans $B_{R} \times(0, T)$, pour tout $T>0$.

$$
\begin{cases}(u, \chi) \in L^{\infty}\left(0, \infty ; C^{0}\left(\bar{B}_{R}\right)\right) \times L^{\infty}\left(B_{R} \times(0, \infty)\right) \\ \Delta u(\cdot, t)=0 & \text { dans } B_{R}-B_{r(t)}, t>0 \\ u(\cdot, t)=M & \text { sur } \partial B_{R}, t>0 \\ u(\cdot, t)=0 & \text { dans } B_{r(t)}, t>0 \\ \chi=c+(1-c) \chi(\{u>0\}) . & \end{cases}
$$

Démonstration. - En premier lieu nous devons démontrer que $u \in$ $L^{2}\left(0, T ; H^{1}\left(B_{R}\right)\right)$ pour tout $T>0$. Puisque $u(\cdot, t) \equiv M$ pour $t>T_{*}$ il suffit de s'assurer que $u \in L^{2}\left(0, T_{*} ; H^{1}\left(B_{R}\right)\right)$. Par définition de $u$ nous avons pour $0<t<T_{*}$ :

$$
\int_{B_{R}}|\nabla u(\cdot, t)|^{2} \mathrm{~d} x \leq \int_{B_{R}}|\nabla v|^{2} \mathrm{~d} x
$$

pour toute fonction $v \in H^{1}\left(B_{R}\right)$ telle que $v=0$ dans $B_{r(t)}$ et $v=M$ sur $\partial B_{R}$. En choisissant $v(x)=M(|x|-r(t))^{+} /(R-r(t))$, quand $t \rightarrow 0$ nous avons

$$
\int_{B_{R}}|\nabla u(\cdot, t)|^{2} \mathrm{~d} x \leq \frac{M^{2}\left|B_{1}\right|\left(R^{n}-r^{n}(t)\right)}{|R-r(t)|^{2}}=\mathrm{O}\left(t^{-\frac{1}{2}}\right)
$$


$\mathrm{du}$ fait de (3.4). Puisque $t \rightarrow \int_{B_{R}}|\nabla u(\cdot, t)|^{2} \mathrm{~d} x$ est régulière pour $0<t<T_{*}$, nous avons $u \in L^{2}\left(0, T_{*} ; H^{1}\left(B_{R}\right)\right)$.

Considérons maintenant la fonction $\chi$.

Il est évident que $\chi \in L^{\infty}\left(B_{R}(0, \infty)\right)$. De plus, si $0<t<\tau$, nous avons

$$
\begin{aligned}
\|\chi(\cdot, t)-\chi(\cdot, \tau)\|_{L^{1}\left(B_{R}\right)} & =(1-c)\left|B_{r(t)}-B_{r(\tau)}\right| \\
& =(1-c)\left|B_{1}\right|\left(r^{n}(\tau)-r^{n}(t)\right) .
\end{aligned}
$$

Étant donné que $r \in C^{0}([0, \infty[)$ et $r(0)=R$ nous obtenons que $\chi \in C^{0}\left(\left[0, \infty\left[; L^{1}\left(B_{R}\right)\right)\right.\right.$, où $\chi(\cdot, 0)$ est défini par $\chi(\cdot, 0) \equiv c$.

Étant donné que (2.4) est évident nous vérifions (2.5) et (2.6). Soit

$$
w(\rho, s)=\frac{\int_{s}^{\rho} \tau^{1-n} \mathrm{~d} \tau}{\int_{s}^{R} \tau^{1-n} \mathrm{~d} \tau} \quad \text { pour } 0<s<\rho<R
$$

Alors $u$ est donnée par

$$
u(x, t)= \begin{cases}M w(|x|, r(t)) & \text { si } 0<t<T_{*} \text { et } x \in B_{R}-B_{r(t)} \\ 0 & \text { si } 0<t<T_{*} \text { et } x \in B_{r(t)} \\ M & \text { si } t \geq T_{*} \text { et } x \in B_{R} .\end{cases}
$$

De plus, si $0<t<T_{*}$, nous avons

$$
\begin{aligned}
r^{\prime}(t) & =-1-\frac{M}{1-c} \partial_{\rho} w(r(t), r(t)) \\
& =-1-\frac{1}{1-c} \nabla u(x, t) \cdot \frac{x}{|x|}
\end{aligned}
$$

Soit alors $Q^{\prime}=B_{R} \times(0, \infty)$ et soit $v \in H_{0}^{1}\left(Q^{\prime}\right)$ non négative avec supp $v$ borné. En utilisant la fonction $\psi$ donnée par (3.2), inverse de la restriction de $r \grave{a}\left[0, T_{*}\right]$, nous obtenons 


$$
\begin{aligned}
\int_{Q^{\prime}} & \left(-\chi \partial_{t} v+(\nabla u+\chi e) \cdot \nabla v\right)= \\
= & \int_{B_{R}} \mathrm{~d} x \int_{0}^{\infty}\left(-\chi \partial_{t} v\right) \mathrm{d} t+\int_{0}^{\infty} \mathrm{d} t \int_{B_{R}}(\nabla u+\chi e) \cdot \nabla v \mathrm{~d} x \\
= & \int_{B_{R}} \mathrm{~d} x \int_{0}^{\psi(|x|)}\left(-c \partial_{t} v\right) \mathrm{d} t+\int_{B_{R}} \mathrm{~d} x \int_{\psi(|x|)}^{\infty}\left(-\partial_{t} v\right) \mathrm{d} t+ \\
& +\int_{0}^{T *} \mathrm{~d} t \int_{B_{r(t)}} c e \cdot \nabla v \mathrm{~d} x+\int_{0}^{T_{*}} \mathrm{~d} t \int_{B_{R}-B_{r(t)}}(\nabla u+e) \cdot \nabla v \mathrm{~d} x+ \\
& +\int_{T_{*}}^{\infty} \mathrm{d} t \int_{B_{R}} e \cdot \nabla v \mathrm{~d} x \\
= & (1-c) \int_{B_{R}} v(x, \psi(|x|)) \mathrm{d} x+c \int_{0}^{T *} \mathrm{~d} t \int_{\partial B_{r(t)}} e \cdot \frac{x}{|x|} v \mathrm{~d} s+ \\
& +\int_{0}^{T_{*}} \mathrm{~d} t \int_{\partial B_{r(t)}}(\nabla u+e) \cdot\left(-\frac{x}{|x|}\right) v \mathrm{~d} s \\
= & (1-c) \int_{0}^{R} \mathrm{~d} \rho \int_{\partial B_{\rho}} v(x, \psi(\rho)) \mathrm{d} s+ \\
& +\int_{0)}^{T *} \mathrm{~d} t \int_{\partial B_{r(t)}}((c-1) e-\nabla u) \frac{x}{|x|} v \mathrm{~d} s .
\end{aligned}
$$

où nous avons utilisé la formule générale

$$
\int_{B_{R}} \varphi=\int_{0}^{R} \mathrm{~d} \rho \int_{\partial B_{\rho}} \varphi \mathrm{d} s
$$

Avec le changement de variable $\rho=r(t)$ dans une intégrale et l'inégalité de Schwarz dans l'autre, nous obtenons

$$
\begin{aligned}
\int_{Q^{\prime}}\left(-\chi \partial_{t} v+(\nabla u+\chi e) \cdot \nabla v\right) \geq & (c-1) \int_{0}^{T *}\left(\int_{\partial B_{r(t)}} v(x, t) \mathrm{d} s\right) r^{\prime}(t) \mathrm{d} t+ \\
& +\int_{0}^{T_{*}} \int_{\partial B_{r(t)}}\left((c-1)-\nabla u \cdot \frac{x}{|x|}\right) v \mathrm{~d} s
\end{aligned}
$$

qui vaut 0 d'après $(3.8)$. 
Pour démontrer (2.6) considérons $v \in L^{2}\left(0, \infty ; H_{0}^{1}\left(B_{R}\right)\right)$ non négative et à support borné. Alors

$$
\begin{aligned}
\int_{Q^{\prime}} \nabla u \cdot \nabla v & =\int_{0}^{T *} \mathrm{~d} t \int_{B_{R}-B_{r(t)}} \nabla u \cdot \nabla v \mathrm{~d} x \\
& =\int_{0}^{T *} \mathrm{~d} t \int_{\partial B_{r(t)}} v \nabla u \cdot\left(-\frac{x}{|x|}\right) \mathrm{d} s \leq 0
\end{aligned}
$$

puisque $\Delta u=0$ et $u>0$ dans $B_{R}-B_{r(t)}$ et $u=0$ dans $B_{r(t)}$.

Nous sommes, maintenant, en mesure de démontrer le résultat suivant.

THÉORÈME 3.5. - Soient $(u, \chi)$ une solution de (0.5)-(0.9) correspondant à des données $g$ bornées, $B_{R}$ une boule de $\Omega, t_{0} \in[0, T[$ et $c \in[0,1[$ tels que

$$
\chi\left(\cdot, t_{0}\right) \leq c \text { dans } B_{R} .
$$

Alors pour tout $\theta \in(0,1)$ il existe $\delta>0$ tel que

$$
u=0 \text { et } \chi \leq c \quad \text { dans } B_{\theta R} \times\left(t_{0}, t_{0}+\delta\right) .
$$

Démonstration. - Nous pouvons, sans perte de généralité, prendre $t_{0}=0$. D'après la proposition $1.3 u$ est bornée. Pour $M>\|u\|_{L^{\infty}\left(B_{R} \times(0, T)\right)}$ et $\epsilon>0$, nous appelons $\left(u_{M}, \chi_{M}\right)$ la sursolution donnée par la proposition 3.4 et nous posons $u_{M \epsilon}(\cdot, t)=u_{M}(\cdot, t+\epsilon), \chi_{M \epsilon}(\cdot, t)=\chi_{M}(\cdot, t+\epsilon)$. Il est évident que $\left(u_{M \epsilon}, \chi_{M \epsilon}\right)$ est encore une sursolution. D'autre part, on vérifie aisément que $\chi_{M \epsilon}(\cdot, 0) \geq c \geq \chi(\cdot, 0)$ dans la boule $B_{R}$ et qu'il existe $R^{\prime} \in(0, R)$ (qui dépend de $\epsilon$ ) tel que

$$
u_{M \epsilon}>\|u\|_{L^{\infty}\left(B_{R} \times(0, T)\right)} \geq u \text { dans }\left(B_{R}-B_{R^{\prime}}\right) \times(0, T) .
$$

Nous pouvons donc appliquer le théorème 2.8 et obtenir $u \leq u_{M \epsilon}$ et $\chi \leq \chi_{M \epsilon}$ dans $B_{R} \times(0, T)$. En faisant tendre $\epsilon$ vers 0 nous avons

$$
u \leq u_{M} \text { et } \chi \leq \chi_{M} \text { dans } B_{R} \times(0, T),
$$

d'où le résultat.

Remarque 3.6. - En faisant tendre $M$ vers $\|u\|_{L^{\infty}\left(B_{R} \times(0, T)\right)}$ il est évident que (3.11) est encore vrai à la limite. 
Remarque 3.7. - Les démonstrations des théorèmes 2.8 et 3.5 tiennent compte du fait que $(u, \chi)$ est une solution bornée de $(0.2)$ dans $B_{R} \times(0, T)$ : en effet le fait que $(u, \chi)$ soit solution de (0.2)-(0.9) avec $g$ bornée ne s'utilise que pour garantir que $u \in L^{\infty}\left(B_{R} \times(0, T)\right)$. Le théorème 3.5 est donc encore vrai si $(u, \chi)$ est une solution localement bornée de $(0.2)$ sur $\Omega \times(0, T)$ quand $\Omega$ n'est pas borné.

À ce propos nous pouvons dire que si $\Omega$ n'est pas borné il peut exister des solutions non bornées. Par exemple, si l'on prend $\Omega=(0,1) \times(-\infty, 0)$, $\Gamma_{D}=(0,1) \times\{0\}, g=1$ et $\chi^{0} \equiv 1$, tout couple $(u, \chi)$ de la forme $u(x)=1-k(t) e \cdot x$ et $\chi \equiv 1$, avec $k \in L^{2}(0, T)$ non négative, est une solution au sens des fonctions $L^{2}\left(0, T ; H_{\text {loc }}^{1}(\bar{\Omega})\right)$.

Le théorème 3.5 dit que la vitesse de propagation de la saturation $\chi$ est finie et donc la vitesse de propagation de la pression est finie quand le milieu n'est pas saturé (c'est-à-dire $\chi \leq c<1$ ). Par contre si le milieu est saturé la vitesse de propagation de la pression peut être infinie, comme le souligne, plus loin, la remarque 3.9.

Proposition 3.8. - Soient $\Omega=\Omega^{\prime} \times\left(y_{*}, 0\right)$, avec $y_{*}<0$ et $\Omega^{\prime}$ ouvert borné de $\mathbb{R}^{n-1}$, et $\Gamma_{D}=\Omega^{\prime} \times\{0\}$. Soient $T>\left|y_{*}\right|, y^{\prime} \in\left(y_{*}, 0\right), c \in(0,1)$, $g \equiv 0$ et $\chi^{0}=c \chi\left(\left\{y^{\prime}<e \cdot x<0\right\}\right)$. Alors la solution $(u, \chi)$ de $(0.5)-(0.9)$ est donnée par

$$
\begin{gathered}
u(x, t)= \begin{cases}y_{*}+\frac{c}{1-c}\left(t-t_{*}\right)-e \cdot x & \text { dans } Q^{\prime \prime} \\
y^{*}-e \cdot x & \text { dans } Q^{\prime \prime \prime} \\
0 & \text { dans } Q-\left(Q^{\prime \prime} \cup Q^{\prime \prime \prime}\right)\end{cases} \\
\chi(x, t)= \begin{cases}c & \text { dans } Q^{\prime} \\
1 & \text { dans } Q^{\prime \prime} \cup Q^{\prime \prime \prime} \\
0 & \text { dans } Q-\left(Q^{\prime} \cup Q^{\prime \prime} \cup Q^{\prime \prime \prime}\right)\end{cases}
\end{gathered}
$$

où

$$
\begin{aligned}
Q^{\prime} & =\left\{(x, t) \in Q \mid y^{\prime}<e \cdot x+t<0, \tau\left(e \cdot x-y_{*}\right)>\left(y^{*}-y_{*}\right)\left(t-t_{*}\right)\right\} \\
Q^{\prime \prime} & =\left\{(x, t) \in Q \mid t<t^{*}, \tau\left(e \cdot x-y_{*}\right)<\left(y^{*}-y_{*}\right)\left(t-t_{*}\right)\right\} \\
Q^{\prime \prime \prime} & =\left\{(x, t) \in Q \mid e \cdot x<y^{*}, t>t^{*}\right\} \\
y^{*} & =y_{*}-c y^{\prime} \\
t_{*} & =y^{\prime}-y_{*} \\
t^{*} & =-y^{*} \\
\tau & =t^{*}-t_{*}=(c-1) y^{\prime}
\end{aligned}
$$




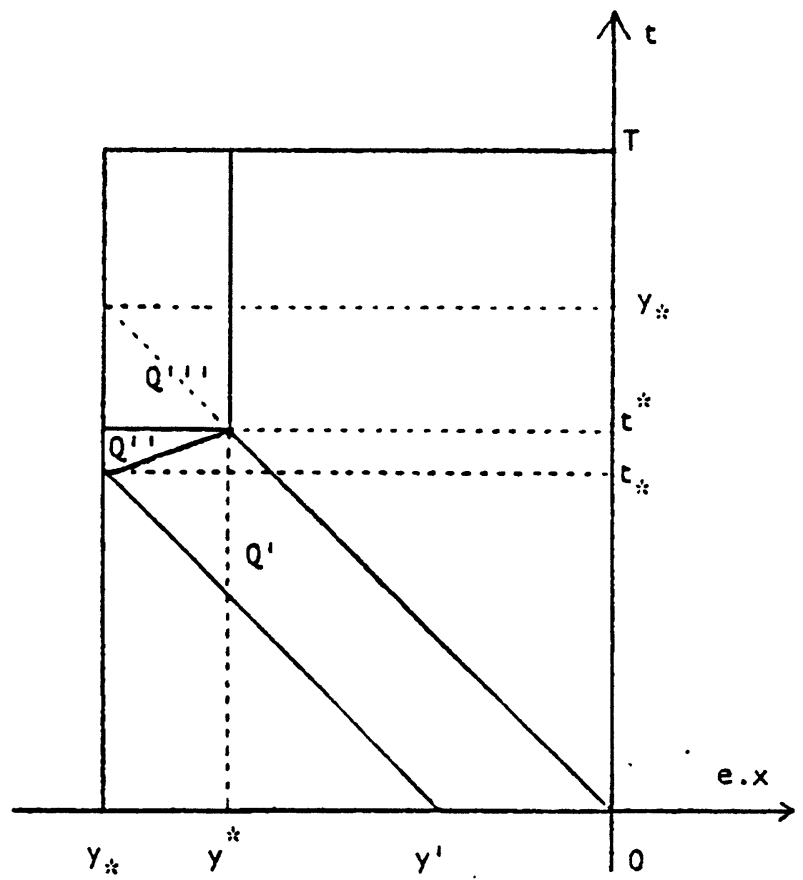

Démonstration. - Il suffit de vérifier (0.9). Soit $v$ comme dans $(0.9)$ et soient $\nu^{\prime}, \nu^{\prime \prime}$ et $\nu^{\prime \prime \prime}$ les vecteurs normaux unitaires de $\partial Q^{\prime}, \partial Q^{\prime \prime}$ et $\partial Q^{\prime \prime \prime}$ orientés vers l'extérieur. Nous avons

$$
\begin{aligned}
& \int_{Q}\left(-\chi \partial_{t} v+(\nabla u+\chi e) \cdot \nabla v\right)= \\
& =c \int_{Q^{\prime}}\left(e \cdot \nabla v-\partial_{t} v\right)-\int_{Q^{\prime \prime}} \partial_{t} v-\int_{Q^{\prime \prime \prime}} \partial_{t} v \\
& =c \int_{\partial Q^{\prime}} v \nu^{\prime} \cdot(e,-1)-\int_{\partial Q^{\prime \prime}} v \nu^{\prime \prime} \cdot(0,1)-\int_{\partial Q^{\prime \prime \prime}} v \nu^{\prime \prime \prime} \cdot(0,1) \\
& =c \int_{\partial Q^{\prime} \cap \partial Q^{\prime \prime}} v \frac{((c-1) e, c)}{\left((c-1)^{2}+c^{2}\right)^{1 / 2}} \cdot(e,-1) \\
& \quad-\int_{\partial Q^{\prime \prime} \cap \partial Q^{\prime}} v \frac{-((c-1) e, c)}{\left((c-1)^{2}+c^{2}\right)^{1 / 2}} \cdot(0,1) \\
& \quad-\int_{\partial Q^{\prime \prime} \cap \partial Q^{\prime \prime \prime}}(0,1) \cdot(0,1) v-\int_{\partial Q^{\prime \prime \prime} \cap \partial Q^{\prime \prime}}(0,-1) \cdot(0,1) v=0
\end{aligned}
$$

ce qui achève la démonstration. 
Remarque 3.9. - Le théorème 3.5 peut être appliqué à la situation précédente avec une boule quelconque et $t_{0}<t_{*}$ (pour simplifier). Dans ce cas on voit que la seconde inégalité de (3.11) est optimale au sens suivant : $\chi$ ne prend que les valeurs $c$ et 1 , comme $\chi_{M}$. Toujours dans le même cas, la vitesse de propagation de la frontière libre vaut $c /(1-c)$.

Dans le cas limite, où $c=1$, la vitesse de propagation de la frontière libre est infinie et la pression est discontinue.

On peut aisément voir que la solution correspondant au cas $c=1$ est exactement la limite quand $c \rightarrow 1$ des solutions considérées : elle est donc donnée par (3.12), (3.13) où $Q^{\prime \prime}$ devient vide et $c=1$, c'est-à-dire

$$
\left\{\begin{array}{l}
u(x, t)=\left(y^{*}-e \cdot x\right)^{+} H\left(t-t_{*}\right), \\
\chi(x, t)=\chi\left(\left\{x \in \Omega|a(t)<e \cdot x<a(t)+| y^{\prime} \mid\right\}\right),
\end{array}\right.
$$

où $a(t)=\max \left(y^{\prime}-t, y_{*}\right)$.

On voit bien que $u$ est discontinue, même si nous la considérons comme une fonction de $(0, T)$ à valeurs dans $\mathcal{D}(\Omega)$. De plus $\chi$ n'est pas la fonction caractéristique de $\{u>0\}$.

Remarque 3.10. - Observons que (3.14) devient stationnaire en temps fini.

\section{Références}

[1] ARonson (D.G.) . - Some properties of the interface for a gas flow in porous media,

Free boundary problems : theory and applications, vol. 1, Research Notes in Mathematics, 78 (1983) pp. 150-159.

[2] CARrillo (J.) .- Uniqueness of the solution of the evolution dam problem, à paraître.

[3] Diaz (J.I.) .- Solutions with compact support for some degenerate parabolic problems,

Nonlinear Analysis, vol. 3, 6 (1979) pp. 831-847.

[4] Dibenetto (E.) and Friedman (A.) . - Periodic behaviour for the evolutionary dam problem and related free boundary problems, Comm. P.D.E., 11 (1986) pp. 1297-1377.

[5] Friedman (A.) Torelli (A.).- A free boundary problem connected with non steady filtration in porous media, Nonlinear Analysis, 1 (1977) pp. 503-545. 
[6] Gilardi (G.) . - A new approach to evolution free boundary problems, Comm. P.D.E., 4 (1979) pp. 1099-1123, 5 (1980) pp. 983-984.

[7] KRÖNER (D.) . - Large time behaviour for the solution of the nonsteady dam problem, in Variational methods for free surfaces interfaces, Concus \& finn ed., Springer (1985).

[8] TORELLI (A.). - On a free boundary problem connected with non steady filtration phenomenon, Ann. Sc. Norm. Sup. Pisa, (4) 8 (1977) pp. 33-59.

[9] Visintin (A.) . - Existence results for some free boundary filtration problems, Ann. Mat. Pura Appl., (4) 124 (1980) pp. 320-393. 